\title{
New records and an updated checklist of lichenicolous fungi from India
}

\author{
Joshi $Y^{1}$, Upadhyay $S^{1}$, Shukla $S^{1}$, Nayaka $S^{2}$ and Rawal $R^{3}$ \\ ${ }^{1}$ Lichenology laboratory, Department of Botany, S.S.J. Campus, Kumaun University, Almora - 263601, Uttarakhand, \\ Indiadryogeshcalo@gmail.com \\ ${ }^{2}$ Lichenology laboratory, National Botanical Research Institute, Rana Pratap Marg, Lucknow - 226001, Uttar \\ Pradesh, India \\ ${ }^{3}$ G.B. Pant Institute of Himalayan Environment and Development, Kosi-Katarmal, Almora - 263643, Uttarakhand, \\ India
}

Joshi Y, Upadhyay S, Shukla S, Nayaka S, Rawal RS 2015 - New records and an updated checklist of lichenicolous fungi from India. Mycosphere 6(2), 195-200, Doi 10.5943/mycosphere/6/2/9

\begin{abstract}
The present paper describes three new records of lichen inhabiting fungi belonging to the genera Biatoropsis, Homostegia and Lichenodiplis, and also report new hosts for Homostegia hertelii and range extensions of Lichenodiplis lecanorae, Pyrenidium actinellum and Sphinctrina tubaeformis in India. An updated checklist of all lichenicolous fungi known so far from India is also provided.
\end{abstract}

Key words - Biodiversity - checklist - distribution - mycobiota - parasymbiont

\section{Introduction}

Lichens are known to host a wide range of microorganisms including lichenicolous fungi that are parasitic fungi living on lichens. They are most commonly specific to a given fungus as the host, but also include a wide range of pathogens, saprotrophs and commensals. In the world so far about 1800 species of lichenicolous fungi have been identified and an occurrence of more than 3000 is being estimated (Lawrey \& Diederich 2011). In India, lichenicolous fungi were hardly studied by lichenologists and the literature is scattered and scanty. Recently, Zhurbenko (2013) published the first list of lichenicolous fungi for India with 42 species including some taxa identified only up to generic level. However, few species of lichenicolous fungi reported earlier from India were missing in Zhurbenko's (2013) list, while, few are discovered recently. The list of missing fungi includes - Arthonia diorygmae S. Joshi \& Upreti (Joshi et al. 2013), Carbonea vitellinaria (Nyl.) Hertel (Awasthi 1991), Sphinctrina anglica Nyl. and Sphinctrina tubaeformis A. Massal. (Pant \& Awasthi 1989). Recently, Joseph and Sinha (unpbl.) reported two species of Melaspilea (M. amarkantakensis S. Joseph \& G.P. Sinha and M. insitiva Stirt.) as lichenicolous fungi from India. In the present communication, we are reporting six interesting species of the lichenicolous fungi based on fresh collections from Kumaun Himalaya and herbarium specimen lodged at National Botanical Research Institute (LWG). Out of six species, three are new to India, while five are new to Uttarakhand (including two new records for India), and described in brief along with their host species and distribution within and outside India. After including the species missed out by Zhurbenko (2013) along with recent records, the total tally of lichenicolous fungi in 
India raises up to 51 (Table 1). This updated checklist would act as baseline information for further studies on lichenicolous fungi of India.

\section{Materials \& Methods}

The study is based on 11 samples collected from Kumaun Himalaya during 2013-14 and one herbarium specimen deposited at LWG. The external morphology of the samples was examined under stereozoom microscope (OLYMPUS SZ2-ILST). The anatomical studies were carried out using hand cut sections using CX21iLEDFS1 microscope. Studied specimens are deposited in the herbarium of Kumaun University, Almora (ALM) and CSIR-National Botanical Research Institute, Lucknow (LWG).

\section{Results}

Biatoropsis usnearum Räsänen, Ann. Bot. Soc. Zool.-Bot. Fenn. "Vanamo" 5: 8 (1934).

Basidiomata club shaped, flat to convex, yellowish to brownish, constricted at base or subsessile, rarely with prominent short stalk. Hymenium hyaline, basidia clavate, up to 3 septate, 25-30 $\times 3-5 \mu \mathrm{m}$. Basidiospores subglobose to ellipsoidal, 4-7 × 3.5-7.5 $\mu \mathrm{m}$.

Known distribution - Cosmopolitan, known from all continents except Antarctica (Diederich 2004a). New to India.

Material examined - INDIA, Tamil Nadu, Kanya Kumari, Upper Kodayar, alt. 1050 m, on thallus of corticolous Usnea austroindica, May 2011, Ravichandran, s.n. (LWG).

Homostegia hertelii D. Hawksw., V. Atienza \& M.S. Cole, Biblioth. Lichenol. 88: 189 (2004).

Stroma arising on the surface of the host thallus, black, matt to slightly shiny, orbicular to irregular. Perithecioid locules arising deep in the stroma. Hamathecium well developed. Asci elongate to clavate, 8 spored. Ascospores 3 septate, olivaceous brown, the tips of the end cells often somewhat paler to subhyaline, smooth walled, 20-27.5 ×7-13.5 $\mu \mathrm{m}$.

Known distribution - U.S.A. (Hawksworth et al. 2004). New to Asia and India.

Material examined - INDIA, Uttarakhand, Almora district, Jhakar Saim Forest, on thallus of Flavoparmelia caperata and Punctelia rudecta colonizing Quercus tree, 06 July 2014, M. Tripathi, K. Chandra \& S. Upadhyay, s.n. (ALM); Dol Ashram, on thallus of Punctelia rudecta colonizing Quercus tree, 15 March 2014, M. Tripathi \& party, s.n. (ALM).

Lichenodiplis lecanorae (Vouaux) Dyko \& D. Hawksw., Lichenologist 11(1): 52 (1979).

Pycnidia present, arising singly, dark brown, subglobose, 50-120 $\mu \mathrm{m}$ in diameter. Conidiophores absent, conidiogenous cells hyaline to pale brown, lageniform to subcylindrical, 5.5-12 $\times 2-3 \mu \mathrm{m}$. Conidia pale brown, 1 septate, elongate to ellipsoid, 4-7.5 $\times 2-3 \mu \mathrm{m}$.

Known distribution - Europe, North and South America, possibly cosmopolitan (Diederich 2004b). Previously the species was reported by Zhurbenko (2013) from Jammu \& Kashmir, where it was colonizing on apothecia and thallus of Caloplaca cerina and Xanthoria candelaria respectively. It is new to Uttarakhand and found growing on apothecial discs of Lecanora species.

Material examined - INDIA, Uttarakhand, Almora district, G.B. Pant Institute of Himalayan Environment and Development, on apothecia of Lecanora sp. colonizing bark of Ligustrum nepalense, 16-18 March 2014, G. Bhakuni, s.n. (ALM).

Lichenodiplis lichenicola Dyko \& D. Hawksw., Lichenologist 11(1): 56 (1979).

Pycnidia present, arising singly, dark brown, subglobose, 50-60 $\mu \mathrm{m}$ in diameter. Conidiophores absent, conidiogenous cells hyaline to pale brown, lageniform to subcylindrical, 5$10 \times 2-3 \mu \mathrm{m}$. Conidia pale brown, 1 septate, elongate to ellipsoid, 9-12 $\times 4-5 \mu \mathrm{m}$.

Known distribution - Alaska (Zhurbenko et al. 1995), British Isles (Coppins \& Coppins 1996), Norway (Hawksworth \& Dyko 1979), Spain (Calatayud et al. 1995). New to Asia and India. 
Table 1 Updated checklist of lichenicolous fungi from India along with their host lichens and distribution

\begin{tabular}{|c|c|c|c|c|}
\hline S. No. & Lichenicolous fungi & Host & Distribution & Reference(s) \\
\hline 1. & Abrothallus peyritschii (Stein) I. Kotte & Vulpicida pinastri & Himachal Pradesh & Alstrup \& Ahti (2007) \\
\hline 2. & Arthonia diorygmae S. Joshi \& Upreti & Diorygma junghuhnii & Tamil Nadu & Joshi et al. (2013) \\
\hline 3. & A. molendoi (Heufl. ex Frauenf.) R. Sant. & Xanthoria elegans & Jammu \& Kashmir & Zhurbenko (2013) \\
\hline 4. & Biatoropsis usnearum Räsänen & Usnea austroindica & Tamil Nadu & This manuscript \\
\hline 5. & Carbonea vitellinaria (Nyl.) Hertel & Candelaria vitellina & Jammu \& Kashmir & Awasthi (1991) \\
\hline 6. & Cercidospora macrospora (Uloth) Hafellner \& Nav.-Ros. & Lecanora sp. & Jammu \& Kashmir & Zhurbenko (2013) \\
\hline 7. & C. melanophthalmae Nav.-Ros., Calat. \& Hafellner & Rhizoplaca melanophthalma & Jammu \& Kashmir & Zhurbenko (2013) \\
\hline 8. & C. xanthoriae (Wedd.) R. Sant. & Xanthoria elegans & Jammu \& Kashmir & Zhurbenko (2013) \\
\hline 9. & Cercidospora sp. & Lecanora sp. & Jammu \& Kashmir & Zhurbenko (2013) \\
\hline 10. & Cladosporium licheniphilum Heuchert \& U. Braun & Xanthoria Candelaria & Jammu \& Kashmir & Zhurbenko (2013) \\
\hline 11. & Corticifraga peltigerae (Fuckel) D. Hawksw. \& R. Sant. & $\begin{array}{l}\begin{array}{l}\text { Peltigera elisabethae } \\
\text { ponojensis }\end{array}\end{array}$ & Jammu \& Kashmir & Zhurbenko (2013) \\
\hline 12. & Endococcus incrassatus Etayo \& Breuss & Endocarpon pusillum & Jammu \& Kashmir & Zhurbenko (2013) \\
\hline 13. & E. rugulosus (Borrer ex Leight.) Nyl. & Rhizocarpon disporum & Jammu \& Kashmir & Triebel (1989) \\
\hline & & Aspicilia $\mathrm{sp}$. & Jammu \& Kashmir & Zhurbenko (2013) \\
\hline 14. & Homostegia hertelii D. Hawksw., V. Atienza \& M.S. Cole & $\begin{array}{l}\text { Flavoparmelia caperata } \\
\text { Punctelia rudecta }\end{array}$ & Uttarakhand & This manuscript \\
\hline 15. & H. piggotii (Berk. \& Broome) P. Karst. & Parmelia sp. & Uttarakhand & Alstrup \& Ahti (2007) \\
\hline 16. & $\begin{array}{l}\text { Intralichen christiansenii (D. Hawksw.) D. Hawksw. \& } \\
\text { M.S. Cole }\end{array}$ & Candelariella aurella & Jammu \& Kashmir & Zhurbenko (2013) \\
\hline 17. & Lichenoconium usneae (Anzi) D. Hawksw. & Flavoparmelia caperata & Jammu \& Kashmir & Zhurbenko (2013) \\
\hline 18. & L. xanthoriae M.S. Christ. & Melanelixia subargentifera & Jammu \& Kashmir & Zhurbenko (2013) \\
\hline 19. & Lichenodiplis lecanorae (Vouaux) Dyko \& D. Hawksw. & $\begin{array}{l}\text { Caloplaca cerina \& Xanthoria } \\
\text { candelaria }\end{array}$ & Jammu \& Kashmir & Zhurbenko (2013) \\
\hline & & Lecanora sp. & Uttarakhand & This manuscript \\
\hline 20. & L. lichenicola Dyko \& D. Hawksw. & Rinodina sp. & Uttarakhand & This manuscript \\
\hline 21. & $\begin{array}{l}\text { Lichenopeltella swaminathaniana Harih., Mibey \& D. } \\
\text { Hawksw. }\end{array}$ & Porina sp. & Tamil Nadu & Hariharan et al. (1996) \\
\hline 22. & $\begin{array}{l}\text { Lichenostigma alpinum (R. Sant., Alstrup \& D. Hawksw.) } \\
\text { Ertz \& Diederich }\end{array}$ & Pertusaria albescens & Jammu \& Kashmir & Zhurbenko (2013) \\
\hline 23. & L. cosmopolites Hafellner \& Calat. & Xanthoparmelia stenophylla & Jammu \& Kashmir & Zhurbenko (2013) \\
\hline 24. & L. cf. elongatum Nav.-Ros. \& Hafellner & $\begin{array}{l}\text { Lecanora sp. \& Lobothallia } \\
\text { praeradiosa }\end{array}$ & Jammu \& Kashmir & $\begin{array}{l}\text { Zhurbenko } \\
\text { (2013) }\end{array}$ \\
\hline 25. & L. subgen. Lichenogramma sp. & Seirophora contortuplicata & Jammu \& Kashmir & Zhurbenko (2013) \\
\hline 26. & $\begin{array}{l}\text { Marchandiomyces corallinus (Roberge) Diederich \& D. } \\
\text { Hawksw. }\end{array}$ & $\begin{array}{l}\text { Physcia aipolia \& Xanthoria } \\
\text { candelaria }\end{array}$ & Jammu \& Kashmir & Zhurbenko (2013) \\
\hline 27. & Melaspilea amarkantakensis S. Joseph \& G.P. Sinha & Pertusaria amarkantakana & Madhya Pradesh & Joseph \& Sinha (unpbl.) \\
\hline 28. & M. insitiva Stirt. & Pertusaria leioplaca & West Bengal & Joseph \& Sinha (unpbl.) \\
\hline
\end{tabular}




\begin{tabular}{|c|c|c|c|c|}
\hline S. No. & Lichenicolous fungi & Host & Distribution & Reference(s) \\
\hline 29. & Monodictys epilepraria Kukwa \& Diederich & Lepraria sp. & Jammu \& Kashmir & Zhurbenko (2013) \\
\hline 30. & Muellerella erratica (A. Massal.) Hafellner \& Volk. John & Lecidea lapicida \& Lecanora sp. & Jammu \& Kashmir & Zhurbenko (2013) \\
\hline 31. & M. pygmaea (Körb.) D. Hawksw. & $\begin{array}{l}\text { Xanthoria elegans \& Acarospora } \\
\text { sp. }\end{array}$ & Jammu \& Kashmir & Zhurbenko (2013) \\
\hline 32. & Nectriopsis lecanodes (Ces.) Diederich \& Schroers & Peltigera elisabethae \& P. scabrosa & Jammu \& Kashmir & Zhurbenko (2013) \\
\hline 33. & Opegrapha foreaui (Moreau) Hafellner \& R. Sant. & Heterodermia leucomelos & Tamil Nadu & $\begin{array}{l}\text { Coppins \& Kondratyuk } \\
\text { (1998) }\end{array}$ \\
\hline 34. & Phoma sp. & Xanthoria elegans & Jammu \& Kashmir & Zhurbenko (2013) \\
\hline 35. & Phyllosticta galligena Moreau & Parmotrema perforatum & Tamil Nadu & Moreau (1951) \\
\hline 36. & Polycoccum clauzadei Nav.-Ros. \& Cl. Roux. & Xanthoria elegans & Jammu \& Kashmir & Zhurbenko (2013) \\
\hline 37. & P. pulvinatum (Eitner) R. Sant. & Physcia dubia & Jammu \& Kashmir & Zhurbenko (2013) \\
\hline 38. & Pronectria subimperspicua (Speg.) Lowen & Punctelia borreri & Jammu \& Kashmir & Zhurbenko (2013) \\
\hline \multirow[t]{2}{*}{39.} & Pyrenidium actinellum $\mathrm{Nyl}$. & $\begin{array}{l}\begin{array}{l}\text { Peltigera elisabethae } \\
\text { praetextata }\end{array} \\
\text { \& } P .\end{array}$ & Jammu \& Kashmir & Zhurbenko (2013) \\
\hline & & Punctelia rudecta & Uttarakhand & This manuscript \\
\hline 40. & Rosellinula frustulosae (Vouaux) R. Sant.. & Lecanora argopholis & Jammu \& Kashmir & Zhurbenko (2013) \\
\hline 41. & Sarcogyne sphaerospora J. Steiner & Candelariella $\mathrm{sp}$. & Jammu \& Kashmir & Zhurbenko (2013) \\
\hline 42. & Skyttea fusispora Sherwood, D. Hawksw. \& Coppins & Ochrolechia trochophora & Assam & Sherwood et al. (1981) \\
\hline 43. & $\begin{array}{l}\text { Sphaeropezia cf. lecanorae (Diederich \& G. Marson) } \\
\text { Baloch \& Wedin }\end{array}$ & Lecanora muralis & Jammu \& Kashmir & Zhurbenko (2013) \\
\hline 44. & Sphinctrina anglica Nyl. & Pertusaria sp. & Tamil Nadu \& Uttar Pradesh & $\begin{array}{l}\text { Pant \& Awasthi (1989) } \\
\text { and Awasthi \& Singh } \\
\text { (1975) }\end{array}$ \\
\hline \multirow[t]{2}{*}{45.} & S. tubaeformis A. Massal. & Pertusaria sp. & $\begin{array}{l}\text { Assam, Manipur, Tamil } \\
\text { Nadu }\end{array}$ & Pant \& Awasthi (1989) \\
\hline & & & Uttarakhand & This manuscript \\
\hline 46. & Stigmidium gyrophorarum (Arnold) D. Hawksw. & Umbilicaria vellea & Jammu \& Kashmir & Zhurbenko (2013) \\
\hline 47. & S. pumilum (Lettau) Matzer \& Hafellner & Phaeophyscia ciliata & Jammu \& Kashmir & Zhurbenko (2013) \\
\hline 48. & S. tabacinae (Arnold) Triebel & Toninia tristis & Jammu \& Kashmir & Zhurbenko (2013) \\
\hline 49. & Vouauxiella lichenicola (Linds.) Petr. \& Syd. & Lecanora sp. & Jammu \& Kashmir & Zhurbenko (2013) \\
\hline 50. & Zwackhiomyces coepulonus (Norman) Grube \& R. Sant. & Xanthoria elegans & Jammu \& Kashmir & Zhurbenko (2013) \\
\hline 51. & Z. cf. kiszkianus D. Hawksw. \& Miadl. & Peltigera elisabethae & Jammu \& Kashmir & Zhurbenko (2013) \\
\hline
\end{tabular}

Material examined - INDIA, Uttarakhand, Champawat district, Shivalaya temple, on apothecia of Rinodina sp. colonizing rocks, 17 July 2014 , K. Chandra, s.n. (ALM). 
Pyrenidium actinellum Nyl., Flora (Regensburg) 48: 210 (1865).

Gall forming fungi on host thalli. Ascomata perithecioid pseudothecia. Ascomata wall entirely brown to dark brown. Paraphysoids filiform, hyaline, septate. Periphyses hyaline, green pigmented at ostiolar region. Asci cylindrical to clavate, 4-8 spored. Ascospores brown with the tips of the end cells often pale brown to subhyaline, ellipsoid to broadly fusiform, 3 septate, rarely 2 or $4,22.5-25 \times 7.5-10 \mu \mathrm{m}$.

Known distribution - Cosmopolitan (Navarro-Rosinés \& Roux 2007). Previously Zhurbenko (2013) reported the species from Jammu \& Kashmir, where it was colonizing thallus of Peltigera elisabethae and $P$. praetextata. It is new to Uttarakhand and found growing on thallus of Punctelia rudecta, thus extending its range distribution as well as host preference.

Material examined - INDIA, Uttarakhand, Almora district, Syahi Devi Forest, on thallus of Punctelia rudecta colonizing Quercus tree, 27 July 2014, S. Upadhyay \& N. Rana, s.n. (ALM).

Sphinctrina tubaeformis A. Massal., Memor. Lich.: 155 (1853).

Apothecia immersed in the host thallus. Stalk absent to short. Capitulum spherical, shiny black or dark brown. Asci cylindrical. Ascospores ellipsoid with pointed ends, non septate, (9-)12$15.5(-20) \times 6-8(-9) \mu \mathrm{m}$.

Known distribution - Europe, Asia, Africa, North and Central America (Tibell 2004). Previously Pant \& Awasthi (1989) and Singh (1981) reported the species from Assam, Manipur and Tamil Nadu states of India, where it was colonizing thallus of Pertusaria. It is new to Uttarakhand and found growing on thallus of Pertusaria sp., thus extending its range distribution within India.

Material examined - INDIA, Uttarakhand, Almora district, Jhakar Saim Forest, on thallus of Pertusaria colonizing Quercus tree, 06 July 2014, M. Tripathi, K. Chandra \& S. Upadhyay, s.n. (ALM); Syahi Devi Forest, on thallus of Pertusaria colonizing Quercus tree, 27 July 2014, S. Upadhyay \& N. Rana, s.n. (ALM).

\section{Discussion}

The present study raises the tally of lichenicolous fungi up to 51 in India and opens a new horizon to study this group since there are several possibilities of finding new species and new records of lichenicolous fungi from India, because a lot of work has been conducted on lichens of India, but this group has remained untouched. Besides this, this group need to be explored out extensively for their host preference, since they are generally named after the host lichen on which they colonize, but there are some species that colonizes various lichens. For example, Homostegia hertelii and Pyrenidium actinellum were previously reported by earlier workers colonizing Flavoparmelia spp. and Peltigera spp. respectively, but present study extends their host range to Punctelia rudecta.

\section{Acknowledgements}

One of the authors (YJ) would like to thank University Grants Commission [41488/2012(SR)] and Scientific and Engineering Research Board [SB/FT/LS-313/2012] for financial assistance, while another author (SN) would like to thank Dr. Paul Diederich for identifying Biatoropsis usnearum. Thanks are also extended to Director, G.B. Pant Institute of Himalayan Environment and Development for allowing us to collect lichen samples from the institute.

\section{References}

Alstrup V, Ahti T. 2007 - New reports of lichenicolous fungi, mainly from Finland and Russia. Karstenia 47, 1-4.

Awasthi DD, Singh KP. 1975 - Additions to the lichen flora of India - III. Geophytology 5, 110112. 
Awasthi DD. 1991 - A key to microlichens of India, Nepal and Srilanka. Bibliotheca Lichenologica 40, 1-337.

Calatayud V, Atienza V, Barreno E. 1995 - Lichenicolous fungi from the Iberian Peninsula and the Canary Islands. Mycotaxon 55, 363-382.

Coppins BJ, Coppins AM. 1996 - New, rare and interesting British lichen and lichenicolous fungus records. Bulletin British Lichen Society 78, 60.

Coppins BJ, Kondratyuk SY. 1998 - Opegrapha trassii sp. nov., a new lichenicolous fungus on Heterodermia. Folia Cryptogamica Estonica 32, 9-14.

Diederich P. 2004a - Biatoropsis Räsänen (1934). In: Lichen Flora of the Greater Sonoran Desert Region. Vol. 2. (eds TH Nash III, BD Ryan, P Diederich, C Gried, F Bungartz). Lichens Unlimited, Arizona State University, Tempe, Arizona, pp. 633.

Diederich P. 2004b - Lichenodiplis Dyko \& D. Hawksw. (1979). In: Lichen Flora of the Greater Sonoran Desert Region. Vol. 2. (eds TH Nash III, BD Ryan, P Diederich, C Gried, F Bungartz). Lichens Unlimited, Arizona State University, Tempe, Arizona, pp. 661-663.

Hariharan GN, Mibey RK, Hawksworth DL. 1996 - A new species of Lichenopeltella on Porina in India. Lichenologist 28, 294-296.

Hawksworth DL, Dyko BJ. 1979 - Lichenodiplis and Vouauxiomyces: two new genera of lichenicolous coelomycetes. Lichenologist 11, 51-61.

Hawksworth DL, Atienza V, Cole MS. 2004 - Lichenicolous species of Homostegia (Dothideomycetes), with the description of $H$. hertelii sp. nov., a new fungus on Flavoparmelia species. Bibliotheca Lichenologica 88, 187-194.

Joshi S, Upreti DK, Nayaka S. 2013 - A new lichenicolous Arthonia species (Arthoniaceae) on Diorygma from India. Lichenologist 45, 323-327.

Lawrey JD, Diederich P. 2011 - Lichenicolous fungi - worldwide checklist, including isolated cultures and sequences available. http://www.lichenicolous.net (accessed 13 September 2014).

Moreau F. 1951 - Un champignon lichenicole cecidogene, Phyllosticta galligena, sp. nov. Bulletin de la Société Botanique de France 98, 100-102.

Navarro-Rosinés P, Roux Cl. 2007 - Pyrenidium Nyl. (1865). In: Lichen Flora of the Greater Sonoran Desert Region. Vol. 2. (eds TH Nash III, BD Ryan, P Diederich, C Gried, F Bungartz). Lichens Unlimited, Arizona State University, Tempe, Arizona, pp. 404-405.

Nylander W. 1865 - Novitatiae quaedum lichenum Europaeorum variorum tribuun. Flora 48, 209-213.

Pant G, Awasthi DD. 1989 - Caliciales from India and Nepal. Biovigyanam 15, 3-27.

Sherwood MA, Hawksworth DL, Coppins BJ. 1981 - Skyttea, a new genus of odontotremoid lichenicolous fungi. Transactions of the British Mycological Society 75, 479-490.

Singh KP. 1981 - Microlichens from Manipur, India. Geophytology 11, 242-256.

Tibell L. 2004 - Sphinctrina Fr. (1825). In: Lichen Flora of the Greater Sonoran Desert Region. Vol. 2. (eds TH Nash III, BD Ryan, P Diederich, C Gried, F Bungartz). Lichens Unlimited, Arizona State University, Tempe, Arizona, pp. 699-701.

Triebel D. 1989 - Lecideicole Ascomyceten. Eine Revision der obligat lichenicolen Ascomyceten auflecideoiden Flechten. Bibliotheca Lichenologica 35, 1-278.

Zhurbenko MP, Santesson R, Walker DA, Auerbach NA, Lewis B. 1995 - New and interesting lichenicolous fungi and lichens from Alaska. Evansia 12, 92-97.

Zhurbenko MP. 2013 - A first list of lichenicolous fungi from India. Mycobiota 3, 19-34. 